\title{
EL LUGAR DEL ESTADO EN LA INTEGRACIÓN EUROPEA*
}

\author{
ALBERTO PÉREZ CALVO \\ Catedrático de Derecho Constitucional \\ Universidad de Salamanca
}

SUMARIO

I. Los Estados crean y animan a las comunidades Europeas.

II. La naturaleza jurídica de la Unión Europea.

III. La Constitución Europea.

\section{LOS ESTADOS CREAN Y ANIMAN A LAS COMUNIDADES EUROPEAS}

\subsection{LA FUNCIÓN DECISIVA DE LOS ESTADOS EN LA VIDA DE LAS COMUNIDADES Y DE LA UNIÓN}

Aunque en algunos análisis parece olvidarse, todos sabemos que las Comunidades Europeas no nacieron por generación espontánea sino que fueron creadas mediante tratado internacional por varios Estados europeos.

Estos Estados fundadores pretendían crear un artefacto que les permitiera alcanzar ciertos fines que ellos solos, aisladamente, no podían. Se trataba de la paz y de otros valores no menos decisivos y fundamentales para la vida de sus ciudadanos. Valores que habían sido ignorados en la Europa de las dos Guerras Mundiales y en el periodo de entreguerras. No es necesario insistir en ello.

Hay otro aspecto que quiero destacar. A veces se interpreta que cuando los Estados

* El artículo recoge la ponencia presentada por el autor en el congreso de Derecho Comparado, organizado por el Instituto de Investigaciones Científicas de la UNAM (México), del 10 al 15 de Febrero de 2004. 
le llaman los Tratados) que realmente decide la legislación comunitaria es el Consejo de Ministros, la legitimidad de este Derecho derivado nace del carácter democrático de sus autores, los Ministros de los Estados miembros.

En este aspecto, hoy la situación ha cambiado parcialmente desde el momento en que el Parlamento Europeo colabora cada vez más intensamente en la función legislativa con el Consejo de Ministros. Este Parlamento Europeo es elegido por sufragio universal y, por tanto, constituye una fuente de legitimidad democrática propia de la Unión Europea.

Es decir, en el terreno de la legislación de la Unión coinciden dos fuentes de legitimidad, la de los Estados que proporciona el Consejo de Ministros y la del Parlamento Europeo que se acaba de señalar. Sin embargo, las grandes transformaciones de la organización sólo siguen siendo posibles mediante el Tratado y en relación con este instrumento, debe seguir hablándose de una única fuente de legitimidad, la de los Estados contratantes.

Realmente, no sólo las Comunidades no nacieron por generación espontánea sino que tampoco han permanecido suspendidas en el aire sin ningún punto de apoyo. Los Estados miembros han estado manteniendo el edificio comunitario y otorgándole el soplo vital de cada día.

Pero además de esa constante simbiosis entre los Estados y las Comunidades Europeas, aquéllos, los Estados, han ido perfeccionando y acomodando su propia organización autónoma en función de las experiencias habidas y de nuevas necesidades. Los Estados, señores de los Tratados, han hecho de aquellas rudimentarias Comunidades la impresionante organización actual que llamamos Unión Europea. Es cierto que las Instituciones comunitarias como la Comisión y, especialmente, el Parlamento Europeo y el Tribunal de Justicia han elaborado ideas de desarrollo de la organización comunitaria o, en el caso del Tribunal, ha convertido en explícitos principios que estaban contenidos in nuce en el propio concepto de ordenamiento comunitario (primacía del Derecho comunitario, por ejemplo) y ha creado otros (la aplicabilidad directa de ese Derecho y todos los esfuerzos en materia de Derechos fundamentales) muy importantes y, a veces, decisivos para la buena marcha de la organización comunitaria. Pero han sido los Estados, mediante el instrumento del tratado internacional, quienes han podido dar los grandes pasos en la integración europea, tanto en lo concerniente a la admisión de nuevos miembros, como en la profundización del proceso.

\subsection{El impacto de las Comunidades en los Estados miembros}

Realmente, la organización de varios Estados europeos en las Comunidades de los años 1950 supuso ya un cambio importante para los Estados fundantes. Se esperaba sacar un mejor rendimiento a sus recursos y medios y, como todo el mundo sabe, lo consiguieron plenamente. El Estado clásico, mediante el instrumento de las Comunidades Europeas, lograba solucionar graves problemas históricos y ponía las bases para adaptarse a nuevas circunstancias. En una palabra, el Estado era más funcional, es decir, desarrollaba mejor los fines que los ciudadanos le habían encomendado en sus respectivas Constituciones.

Pero los Estados miembros de las nuevas organizaciones iban a sufrir cambios importantes tanto desde el punto de vista estructural como funcional. Iban a convertirse en Estados comunitarios, una nueva manera de ser de esta forma de organización política soberana nacida a caballo entre los siglos XV y XVI. Primero se comprobó la alteración 
crean las Comunidades Europeas, construyen realmente una organización ajena a ellos mismos. Incluso pueden percibirse prejuicios que podrían ser interpretados en el sentido de que, una vez creadas las Comunidades, lo mejor que podría pasar es que los Estados desaparecieran de la vida de la nueva organización. No se cae en la cuenta de que cuando los Estados crean las Comunidades, lo que en realidad hacen es organizarse ellos mismos, y a través de ellos, sus ciudadanos, en una sociedad u organización europea de la que se convierten en miembros desde el mismo momento de la creación. Esa nueva organización europea nace con unos fines que cumplir y con unas instituciones capaces de adoptar decisiones de carácter general o concreto para poder cumplir los fines que se les encomiendan. Es decir, la nueva organización europea tiene un margen de autonomía para poder actuar sobre unos ámbitos materiales concretos como el carbón y el acero, en un primer momento y, más tarde con la creación de la CEE y CEEA, otros ámbitos materiales nuevos. Pero, aún con su autonomía, las Comunidades constituyen ante todo una sociedad u organización de los Estados miembros y de sus respectivos pueblos.

Desde la sagaz visión del Prof. Italiano S. Romano a principios del siglo XX, a una sociedad u organización de este tipo la consideramos un ordenamiento jurídico. O sea, un grupo humano organizado y con la autonomía suficiente como para poder elaborar sus propias normas jurídicas que no son sino una manifestación constante de la propia renovación de la organización que constituyen. En ese momento, por tanto, hay tres organizaciones europeas de este tipo, la CECA, la CEE y la CEEA que, miradas desde un punto de vista jurídico, constituyen otros tantos ordenamientos jurídicos. Años más tarde, tras los cambios introducidos en las tres organizaciones y, especialmente, tras el Acta Unica Europea, podemos considerar que estamos en presencia de una sola organización europea, aunque con matices que ahora no nos interesan, lo que quiere decir, un solo ordenamiento jurídico.

Por tanto, una vez creadas las Comunidades Europeas por los Estados, éstos no sólo no se desentienden de la obra creada sino que contribuyen ordinariamente a su funcionamiento cotidiano.

Quiero destacar fundamentalmente dos aspectos de esa constante participación de los Estados en la vida de las Comunidades europeas. En primer lugar, la presencia de los Ministros de los Estados miembros en el Consejo de Ministros de las Comunidades; en un segundo momento, la función que los Estados desempeñan en la legitimación de las propias Comunidades y de sus actos.

En relación con el consejo de Ministros no creo que haya que insistir mucho en la función legislativa que ha correspondido realmente a esta institución hasta el Tratado de la Unión Europea que es cuando ha comenzado a compartir esa función con el Parlamento Europeo. Y recordemos que el Consejo de Ministros ha estado y sigue estando compuesto por los Ministros de los Estados miembros.

La función de legitimación de las Comunidades Europeas permite a éstas ser aceptadas como legítimas por los ciudadanos europeos. Y, aunque no habría que advertirlo, la única legitimidad posible hoy en nuestras sociedades es la democrática, la que nace del voto de cada uno de los ciudadanos.

Las reglas fundamentales que están en la base de las Comunidades, los Tratados constitutivos, deben su legitimidad democrática al hecho de que son elaborados en el marco de las respectivas Constituciones de los Estados miembros y de que finalmente son ratificados por los Parlamentos de estos Estados.

Después, en la vida diaria de las Comunidades, cuando el único órgano (institución, 
Europeo de Derechos Humanos para recabar soluciones concretas.

En definitiva, una técnica de Derecho comparado auspiciada por los propios Tratados, lo que quiere decir, objeto de un compromiso entre los Estados comunitarios. De esta manera, al mismo tiempo que se iba construyendo la parte más visible de la integración europea, o sea, las Comunidades, de modo paralelo, los Estados tejían una red jurídica en la misma dirección integradora. La actividad del Estado, por tanto, es fundamental tanto cuando actúa a través de los órganos de la Unión como cuando lo hace fuera de ellos. En este sentido, es preciso destacar la línea iuscomparatista avanzada por el Tribunal Constitucional alemán cuando en su sentencia sobre el Tratado de Maastricht exige de la Unión Europea no soluciones idénticas a las alemanas para garantizar los Derechos Fundamentales sino un nivel similar de garantía. Teniendo en cuenta que esta inteligente solución va a servir para todos los Estados miembros, va a obligar al Tribunal de Justicia de las Comunidades Europeas a estar muy atento a lo que se hace en toda Europa en esta materia para poder atender convenientemente esta exigencia.

El ámbito de las Comunidades Europeas se convierte así, como indica R. Legrand en el paraíso del Derecho comparado. Las manifestaciones institucionales de actividades de Derecho comparado que se han indicado no se agotan en sí mismas sino que constituyen un catalizador para la práctica de esta técnica comparatista, en especial desde la ciencia jurídica que ha dedicado grandes esfuerzos al estudio de la integración jurídica europea y a la comparación de soluciones jurídicas a los problemas planteados. Y esto ha sucedido en todas las ramas del Derecho.

Una llamada de atención importante al respecto en el ámbito del Derecho Constitucional ha sido hecha por el Prof. Häberle.

En un primer momento apela a la que llama «doble vía» en la construcción europea. Es decir, una vía es la propia comunitaria en la que se ha de avanzar hacia una naciente Constitución europea. Y la otra vía es la interna, en la que la construcción comunitaria debe constituir también un tema propio de la Constitución de cada país buscando con ello, entre otros objetivos, que los ciudadanos sientan la construcción europea como algo propio. Incluso llega a proponer en este sentido que lo ideal sería la adición a las Constituciones nacionales de los Estados miembros de artículos sobre Europa concertados de antemano. Algo que probablemente no resultaría fácil de realizar si se tiene en cuenta que tales artículos serían fundamentalmente simbólicos, sin contenido material innovador. También propone lo que denomina Derecho Constitucional Común Europeo la construcción consciente de un acervo común de regulaciones constitucionales y de soluciones jurisprudenciales, especialmente las del Tribunal Europeo de Derechos Humanos. Este acervo constitucional común tendría, entre otras finalidades, la de prestar en todo momento soluciones a los operadores jurídicos que en un momento dado necesitaran de ellas. A mi juicio, si bien no de un modo consciente y programado, así se opera en Europa desde hace tiempo no ya sólo en cuestiones concretas que se le pueden presentar a un operador jurídico sino en las grandes empresas de hacer una Constitución. Para no ir demasiado atrás en el tiempo, tan sólo voy a señalar cómo la Constitución española de 1978 debe mucho a las experiencias constitucionales europeas, especialmente a la alemana y a la italiana.

La sociedad comunitaria europea se organizó un día en las Comunidades Europeas para salvaguardar valores comunes y hoy continúa renovando día a día su organización comunitaria a través de la legislación comunitaria ordinaria o, a veces, de modo extraordinario, mediante el tratado, especialmente a partir del Acta Unica Europea. Esta actualiza- 
del equilibrio de los poderes legislativo y ejecutivo, en beneficio de este último lo que constituye un aspecto de lo que se llamó «déficit democrático» de las Comunidades Europeas. Por supuesto el Estado comunitario adquiría una permeabilidad casi absoluta respecto del Derecho creado por la nueva organización. Más tarde el impacto comunitario se percibió en los problemas que iban a surgir en los Estados compuestos en torno a la articulación entre Federación y Estados federados o, en el caso de España, Estado y Comunidades Autónomas. Incluso quedaban afectados conceptos que parecían inmutables como, por ejemplo, el de la Nación, que adquiría nuevos contenidos cuando el Tratado de la Unión Europea introdujo la categoría nueva de ciudadanía europea. Al mismo tiempo, ese mismo Estado, a través de sus representantes en el Consejo de Ministros, participaba en las decisiones comunitarias junto con los demás Estados miembros. De esta manera, el Estado clásico, hasta entonces capaz de actuar libremente en su ámbito, encontraba límites para adoptar decisiones en las materias que se habían encomendado a las Comunidades Europeas. Ya no podía decidir aisladamente sino que debía acompasar la defensa de sus intereses con los demás Estados comunitarios. Es decir, el Estado, no directamente, sino a través de su presencia en las Comunidades Europeas decidiría mancomunadamente en esas cuestiones, o sea de modo comunitario.

Lo dice muy bien el Proyecto de Constitución Europea en su artículo 1.1.: «La Unión... ejercerá, de modo comunitario, las competencias que éstos (los Estados) le transfieran»)

\subsection{UNA INTENSA HOMOGENEIZACIÓN JURÍDICA DE LOS ESTADOS COMUNITARIOS}

El concepto mismo de Estado comunitario, como categoría propia de los Estados miembros de la Unión Europea, implica ya una idea de cierta homogeneización de estos Estados que sufren algunas transformaciones funcionales y estructurales, como se ha indicado someramente. El funcionamiento correcto de la organización creada, hoy la Unión Europea, exige de sus creadores ese cierto grado de homogeneidad. Por ejemplo, todos los Estados han debido prever en sus constituciones cláusulas que permitan la aprobación de los tratados constitutivos por los que se encomienda a las Comunidades Europeas el ejercicio de competencias derivadas de sus respectivas Constituciones.

Pero este no es más que el primer paso en la homogeneización jurídica de los Estados comunitarios. La consecución de los objetivos encomendados a las Comunidades va a exigir ordinariamente un grado de homogeneidad jurídica muy importante. Los propios Tratados comunitarios van a establecer áreas de actividad en la que preconizan una aproximación de legislaciones entre los Estados. Muchos campos de las actividades sociales, como el ámbito mercantil, en general, van a ser objeto de esta técnica que dará sus frutos en regulaciones europeas comunes para el comercio, la vida de las empresas, etc... Lo mismo va a suceder con los llamados pilares no comunitarizados previstos en el Acta Unica Europea y siguientes Tratados. En especial, es importantísima la aproximación de legislaciones en los ámbitos de los pilares de cooperación, especialmente en el tercero, hoy llamado Cooperación Policial y Judicial en Materia Penal. Fundamental ha sido también la labor del Tribunal de Justicia de las Comunidades Europeas cuando ha elaborado el concepto operativo de «convergencias constitucionales de los Estados miembros» o ha acudido a la jurisprudencia del Tribunal 
La pregunta inicial es puramente retórica. La he hecho porque, a pesar de la sencillez de los datos que nos permiten afirmar el carácter de tratados de estos instrumentos jurídicos, en torno a su naturaleza ha circulado una enorme y, a veces, nebulosa ambigüedad. Por ejemplo, no dejan de sorprendernos ciertas expresiones del Tribunal de Justicia de las Comunidades Europeas:

En la sentencia Van Gend \& Loos, de 1963, se afirma que «la Comunidad constituye un nuevo ordenamiento jurídico de Derecho Internacional, en beneficio del cual los Estados han limitado, aunque sea en campos restringidos, sus derechos soberanos». Es perfectamente comprensible y aceptable todo lo que dice el Tribunal salvo, a mi juicio, la referencia a la limitación de «derechos soberanos» de los Estados.

Más tarde, en la sentencia Costa c. Enel, de 1964, se afirma también que, «al constituir una Comunidad de duración ilimitada, dotada de instituciones propias, de personalidad, de capacidad jurídica, de capacidad de representación internacional, y, más particularmente de poderes reales nacidos de una limitación de competencia o de una transferencia de atribuciones de los Estados a la Comunidad, éstos han limitado, aunque en ámbitos restringidos, sus derechos soberanos». Y se añade en otro momento: «la transferencia operada por los Estados, de su ordenamiento jurídico interno en beneficio del ordenamiento jurídico comunitario, de los derechos y obligaciones correspondientes a las disposiciones del Tratado, implica pues una limitación definitiva de sus derechos soberanos contra la cual no puede prevalecer un acto unilateral ulterior incompatible con la noción de la Comunidad».

Son dos las cuestiones que veo dudosas: la limitación de la soberanía y que ésta limitación sea definitiva porque la Comunidad nace con una pretensión de duración ilimitada.

En cuanto al primer punto, no veo que mediante un Tratado se pueda hacer dejación de la soberanía a no ser que se haga de una manera expresa. Otra cosa es que se limite el ejercicio de poderes soberanos. Esto es algo que los Estados hacen constantemente cuando pactan un tratado entre sí. Mientras el Tratado esté vigente implicará derechos y obligaciones para los Estados signatarios lo que supone una limitación en el ejercicio de sus poderes públicos. Y todos los poderes públicos que despliega el Estado pueden ser considerados como ejercicio de la soberanía en un momento dado. Por lo tanto, los Tratados comunitarios, en este aspecto, no tendrían nada de especial.

En cuanto al carácter definitivo de la limitación porque la vocación temporal de la Comunidad sea ilimitada carece de relación una cosa con la otra a no ser que encadenemos el hecho de la duración ilimitada de la Comunidad a que los miembros no puedan abandonarla. Y aunque los Tratados comunitarios no prevean nada al respecto, me pregunto si no es posible apelar al Derecho internacional sobre los Tratados e invocar la posibilidad de denuncia de los mismos y abandonar así las Comunidades. Ciertamente, el abandono habría de hacerse con las negociaciones debidas con los demás miembros. Lo que creo que no se puede hacer es identificar duración ilimitada de la Comunidad con la imposibilidad de su abandono. Siempre queda a los demás miembros la posibilidad de continuar en ella. Y siempre queda a todos la posibilidad de modificar el Tratado y declarar extinguida la Comunidad.

Por si quedaran dudas al respecto, el Proyecto de Constitución europea, aún recogiendo la duración indefinida de la Unión (Artículo III-339), declara expresamente en su art. 59 la posibilidad de retirada voluntaria de la Unión para todo Estado miembro. Y yo no diría que el Proyecto de Constitución Europea innova nada en este aspecto. Simplemente 
ción constante le permite incorporar nuevos valores y fines a su organización, llamada hoy la Unión Europea.

En Europa, como quizás pudiera suceder en otras partes del mundo, especialmente en Iberoamérica, la operación de coincidir en juridificar ciertos valores no resulta difícil debido a que, en general, los mismos forman parte de una común cultura axiológica, cimentada hoy de modo muy especial en los Derechos fundamentales, liberales y sociales, y en la democracia. No me refiero a lo que se ha llamado común «cultura jurídica» europea que sin duda también coadyuva de modo muy importante a hacer más sencilla la construcción europea y que también debe ser tenida en cuenta a estos efectos. Me refiero ahora a algo más profundo que las concretas formas jurídicas como son los valores que se trata de defender mediante esas formas jurídicas, o sea, mediante una organización precisa.

Estas circunstancias permiten fácilmente la labor de comparación antes señalada y el intercambio o préstamo de conceptos y soluciones jurídicas desde los Estados a la Unión Europea o al revés, desde la propia Unión a los Estados. Estos movimientos ocurren también entre los Estados entre sí en momentos históricos concretos, como se ha visto antes en relación con la elaboración de la Constitución española y, día a día, a través de la jurisprudencia constitucional.

Podemos ver, por tanto, cómo a través de diversos caminos se está produciendo una intensa homogeneización jurídica de los Estados comunitarios lo que, sin ninguna duda, va en el sentido de la propia integración europea, y constituye de hecho una de sus manifestaciones.

De todas formas, como se ha recordado, habría que huir de todo tipo de exageración a la hora de expandir esta homogeneización. A mi juicio, hay dos límites a esta operación. Uno de ellos responde a la dificultad objetiva del traslado de categorías jurídicas si no hay concordancia de valores vigentes. Es decir, una norma podrá ser trasladada a otro ámbito social si responde a un valor vigente en este preciso ámbito. El otro puede resultar de la voluntad soberana expresada en la Constitución del Estado en cuestión.

\section{LA NATURALEZA JURÍDICA DE LA UNIÓN EUROPEA}

\subsection{AmbigüEdades en tORno a los Tratados COMUnitarios}

¿Quién podría dudar que los Tratados internacionales constitutivos de las Comunidades Europeas y los que les han sucedido después son precisamente eso, tratados internacionales?

Son tratados por la razón fundamental de que se trata de instrumentos normativos pactados por Estados soberanos y para cuya reforma se necesita el acuerdo unánime de todos ellos así como su ratificación de acuerdo con sus reglas nacionales. Además, los instrumentos aludidos aquí se denominan a sí mismos «tratados». Aunque este argumento no es decisivo porque el propio legislador podría equivocarse sobre la naturaleza jurídica de una norma. Hay ejemplos de ello tanto en Derecho interno como, quizás, en el ámbito comunitario, como se verá más adelante en relación con el proyecto de «Constitución europea». 
allá del objetivo de facilitar la comprensión de lo que quiero expresar. En este sentido, parto de la idea de Constitución que surge de la experiencia de la Revolución de Estados Unidos. De acuerdo con ello, la Constitución es una norma hecha por el poder soberano, el pueblo, que adopta la forma de poder constituyente y que, por ello, es la norma suprema del ordenamiento jurídico estatal. Estos datos formales van a ser decisivos como criterio para saber si estamos o no ante una Constitución. De todas formas, recordemos también la aportación revolucionaria francesa a nuestro concepto actual de Constitución. Según el art. 16 de la Declaración de los Derechos del Hombre y del Ciudadano, la Constitución debe contener, al menos, la garantía de los Derechos fundamentales y establecer la separación de poderes. Hoy a los Derechos contemplados en la Declaración de 1789 habría que añadir los sociales, según opinión mayoritaria de la doctrina, y la separación de poderes quedaría relativizada de modo que sería indispensable, al menos, la independencia del Poder Judicial.

Los elementos materiales de esta visión de la Constitución podrían estar presentes en normas no constitucionales como es el caso de un Tratado, especialmente los comunitarios, pero no podríamos hablar de Constitución si faltara el elemento formal, la acción de un poder constituyente, o sea, en nuestro caso, un pueblo europeo. Después volveré sobre el asunto.

Desde luego, aún sin acudir a la doctrina de Tribunales Constitucionales de los Estados miembros, creo que, de acuerdo con la idea de Constitución que acabo de indicar, los Tratados comunitarios no lo son.

Los instrumentos que están en la base de la Unión Europea por tanto, son Tratados internacionales. Las Comunidades Europeas en el pasado y hoy la Unión se han movido y se mueven en el mundo internacional, con las matizaciones necesarias, obviamente, como enseguida se verá.

\subsection{RELACIONES ENTRE EL ORDENAMIENTO JURÍDICO COMUNITARIO Y LOS ORDENAMIENTOS INTERNOS}

Como se acaba de ver, el ordenamiento jurídico comunitario debe calificarse como internacional. No obstante, se suele afirmar que tiene unas características propias que le hacen singular. Se señalan los siguientes caracteres específicos: la aplicabilidad directa de sus normas de derecho derivado sin necesidad de normas o actos internos de recepción; el hecho de que sus destinatarios son no sólo los Estados sino también los ciudadanos de esos Estados miembros; y la cualidad de imponerse sobre las normas internas cualquiera que sea el rango de éstas, es decir, la primacía.

A mi juicio, los dos primeros caracteres son realmente nuevos en relación con el Derecho internacional clásico: la eficacia directa y la aplicabilidad a los ciudadanos. No estoy tan seguro de que la primacía sea una cualidad que no posea todo el Derecho internacional. Realmente, ¿Podría existir este Derecho si no se aplicara con preferencia al interno?

No es tarea mía ocuparme ahora de ese asunto, pero un razonamiento similar lleva a concluir que no podría existir el Derecho comunitario sin la primacía sobre el Derecho de los Estados miembros. Si no fuera así, la integración europea, de la que el Derecho comu- 
se limita a decir expresamente lo que antes estaba de modo implícito.

Y no acaban aquí las expresiones dudosas y arriesgadas del Tribunal de Justicia. En su Dictamen 1/91 sobre el Espacio Económico Europeo, el Tribunal dice que «el Tratado CEE, aunque haya sido celebrado en forma de Convenio internacional, no por ello deja de ser la carta constitucional de una Comunidad de Derecho. Conforme a reiterada jurisprudencia del Tribunal de Justicia, los Tratados comunitarios han creado un nuevo ordenamiento jurídico a favor del cual los Estados han limitado, en ámbitos cada vez más amplios, sus derechos de soberanía». La misma expresión se repite en su Sentencia Los Verdes contra el Parlamento Europeo, de 23 de abril de 1986.

Realmente no me parece apropiado que se utilice la expresión «carta constitucional» para calificar a un Tratado. Puede pensarse, y así lo han intentado varios autores, que el tribunal no ha querido exactamente utilizar el adjetivo constitucional en su sentido primigenio y usual en la Ciencia del Derecho. Que lo que ha pretendido es expresar la posición suprema de los Tratados en el ordenamiento comunitario. Pero es difícil mantener esa interpretación. Cuando el Tribunal utiliza la expresión «carta constitucional» lo hace después de la frase concesiva «aunque (el Tratado) haya sido celebrado en forma de Convenio internacional». El Tribunal ve la dificultad que para calificar al texto como constitucional supone el hecho de que haya sido celebrado en forma de Convenio internacional pero, a pesar de todo, lo afirma. Por otra parte, la pretensión constante del Tribunal por considerar al Derecho comunitario dotado de primacía incluso sobre las constituciones de los Estados miembros puede dar verosimilitud a la otra pretensión de calificar como constitucionales a los tratados comunitarios y a las reiteradas afirmaciones sobre la limitación de algunos de sus derechos soberanos.

Otros autores, a través de diversos puntos de vista han considerado a los Tratados comunitarios como una Constitución. Entre estos puntos de vista suele predominar la idea de la función que cumplen estos Tratados: crean un auténtico poder público comunitario y constituyen la regla fundamental del mismo y el criterio de la validez del Derecho derivado y aún el de los Estados miembros. Incluso, el Tribunal de Justicia comunitario cumple funciones similares a las del Juez de la Constitucionalidad de un Estado...

Y ciertamente todas estas afirmaciones son verdaderas. Esas y otras son las funciones que realizan los Tratados. Y también las Constituciones y, en muchas ocasiones, las leyes. Muchas normas jurídicas cumplen funciones similares de organización de poderes públicos y de criterio de validez respecto de las normas de rango inferior y no por eso son llamadas constitucionales. Cierto es que estas normas que se acaban de citar, si tienen el rango de ley, no son supremas respecto del ordenamiento que crean y por encima tienen a la Constitución. Pero tampoco los Tratados pueden imponerse a las Constituciones de los Estados de tal modo que, como sucede en España con todo tipo de Tratados (art. 96CE), en caso de conflicto con la Constitución, ésta debe ser modificada para poder ratificar el Tratado. Es la única manera de hacer compatible el principio de supremacía constitucional con las relaciones internacionales mediante acuerdos. De lo contrario, estos instrumentos internacionales carecerían de la seguridad exigible a una norma jurídica.

De todas formas, ante la autoridad científica de quienes utilizan con tanta naturalidad el adjetivo «constitucional» $\mathrm{o}$, como veremos más adelante, la palabra «Constitución»y, más concretamente, «Constitución Europea», creo conveniente expresar en este momento el concepto de Constitución en que me apoyo. Se trata de una idea sin pretensiones de ir más 
El marco se podría describir de la siguiente manera. Mediante los tratados, al nuevo ordenamiento comunitario se le atribuyen ciertas competencias. A partir de conflictos de normas comunitarias con estatales, el Tribunal de Justicia declara la primacía de las comunitarias de modo que son éstas las que se aplican preferentemente mientras que las internas quedan desplazadas. Por tanto, en caso de conflicto, el Juez comunitario es el único dueño de la decisión mientras que al Estado no le queda ninguna posibilidad ordinaria de dar otra solución (la posibilidad extraordinaria es la reforma de la doctrina del Tribunal mediante la enmienda de los tratados).

En este marco es donde se propone la posibilidad de reforma constitucional que, en relación con decisiones ya adoptadas, podría obviamente solucionar la contradicción cediendo ante la decisión comunitaria. De esta manera, la reforma de la Constitución en este marco de distribución de competencias puede zanjar el problema técnico de la aporía antes señalada, pero no el político, ya que los Estados dejan la iniciativa política en cuestiones fundamentales en manos del instrumento que han creado. El mecanismo propuesto, por tanto, no resulta útil desde el punto de vista de una integración comunitaria democrática que debe ser dirigida fundamentalmente por los Estados miembros en el marco de sus propias Constituciones.

También se ha contemplado la posibilidad de reformas constitucionales para dar cabida a nuevos tratados que hagan avanzar la integración comunitaria.

Así, ante la propuesta de ratificación del Tratado de Maastricht se produjeron una serie de reformas constitucionales en Francia, República Federal de Alemania y Portugal, en las que, entre otros objetivos, se perseguía establecer con la mayor claridad posible los límites a la integración europea o sea, dicho en otras palabras, se pretendía acotar materias o funciones concretas a favor del Estado y por lo tanto, fuera del alcance del Derecho comunitario.

En este sentido son realmente ilustrativas algunas consideraciones de la Sentencia de 12 de octubre de 1993, sobre el Tratado de la Unión Europea, del Tribunal constitucional alemán, en las que reclama para sí la capacidad de controlar el respeto por parte de las Comunidades del ámbito competencial que se les ha atribuido por los Tratados. Y el Tribunal establece el fundamento de su posible control afirmando que las competencias transferidas a las Comunidades Europeas son precisadas «de una forma suficientemente previsible, para que quede preservado el principio de la limitada atribución de competencias especiales, no surja una competencia en materia de competencias para la Unión Europea y la asunción de futuras funciones y competencias por parte de la Unión Europea y las Comunidades Europeas se haga depender de Tratados complementarios y reformas del Tratado[...]». Por todo ello, añade el Tribunal, «si instituciones u órganos europeos dieran en gestionar o desarrollar el Tratado de la Unión de manera que no quedase cubierto por el Tratado en que se funda la Ley de Ratificación, no serían vinculantes en la jurisdicción alemana los actos jurídicos que de él se desprendiesen. Los órganos del Estado alemán tendrían impedimento de índole jurídico-constitucional para aplicar tales actos jurídicos en Alemania. En consecuencia, el Tribunal Constitucional alemán examinaría si los actos jurídicos de instituciones y órganos europeos respetan las fronteras de los derechos de soberanía a ellos otorgados o si, por el contrario, rompen ese marco».

Como puede observarse, la claridad de las observaciones del Tribunal Constitucional alemán es rotunda y acierta de lleno en el diagnóstico del problema. Aunque, aún siendo lógico en el marco aislado del ordenamiento alemán, puede sorprender que el Tribunal 
nitario es una de sus manifestaciones fundamentales, sería imposible como resulta fácil de entender. Cualquier Estado podría esgrimir la excepción de su Derecho interno para librarse de obligaciones comunitarias no queridas. Es decir, la aplicación del Derecho comunitario debe estar garantizada en todos los Estados miembros sin excepción.

Dicho así, sin matices, nos vamos a encontrar inmediatamente con una posible contradicción, la que puede surgir entre el carácter supremo de la Constitución y la que nace de la necesidad de la integración comunitaria. Se trata de la aporía que describe A. López Basaguren entre la primacía del Derecho comunitario y la supremacía de la Constitución. $\mathrm{Y}$, aunque es cierto que en nuestra cultura democrática sólo nos queda aceptar la supremacía de la Constitución, como producto del poder constituyente, lo cierto es que la construcción comunitaria se hace sobre la base de unos Tratados adoptados por los Estados miembros en virtud de una expresa habilitación de sus respectivas Constituciones.

En un primer momento, los Estados, mediante el instrumento de la ratificación, pueden verificar la concordancia de los tratados constitutivos con sus respectivas constituciones. Pero, como recuerda A. López Basaguren, en los primeros años de la integración comunitaria el Tribunal de Justicia jugó un papel central en la dirección del proceso mediante una interpretación expansiva de las competencias comunitarias más allá de lo previsto en los tratados. Especialmente, en relación con los Estados en los que existe un órgano encargado del control de constitucionalidad, se va a producir una distorsión entre la interpretación de este órgano, que tiene como criterio central a la Constitución y la del Tribunal de Justicia, cuyo eje de interpretación son los tratados.

Al mismo tiempo, el Derecho comunitario derivado sólo puede ser controlado, según su propia doctrina, por el Tribunal comunitario cuyos criterios de juicio pueden ser divergentes de los de los órganos internos de Justicia constitucional.

Para solucionar estas posibles contradicciones se han propuesto algunas medidas.

En primer lugar, se ha planteado la posibilidad de modificar la Constitución para romper la mencionada aporía cuando ésta se produzca. Esta medida responde a la lógica del art. 95 de la Constitución española que exige la reforma de la Constitución para poder ratificar un tratado que contenga estipulaciones contrarias a la misma.

Incluso se ha mantenido esta misma solución hacia el futuro, cuando las habilitaciones contenidas en la Constitución en función de la integración europea pueden quedar obsoletas ante los desarrollos comunitarios. En este caso, se trataría de poner al día nuestras Constituciones en función de las nuevas situaciones y necesidades. También se ha avanzado la posibilidad de un procedimiento simultáneo y paralelo de reformas en los distintos Estados miembros que condujeran a textos constitucionales uniformes en esta materia.

De todas formas, ante las posibles dificultades que pueden aparecer en relación con las reformas de la Constitución, se ha planteado también, como una vía de solución complementaria a la anterior, la necesidad de que mediante la interpretación jurídica y desde ambos ordenamientos, el comunitario y los nacionales, se creen reglas de relación entre ellos que puedan ser aceptadas pacíficamente y de forma generalizada desde ambas perspectivas. Estas reglas deberían contemplar los caracteres o elementos que son necesarios para la existencia y funcionamiento del otro ordenamiento (A. López Basaguren).

Para analizar la funcionalidad de las soluciones apuntadas sería necesario tener en cuenta el marco en el que es posible esta contradicción que resulta aparentemente insoluble, o sea, una aporía. 
cisa posible en relación con las competencias que se atribuyen a la Unión Europea y las reglas concernientes a las relaciones interordinamentales.

No entro ahora a valorar si sería más funcional mantener el instrumento de los tratados o intentar aprobar una Constitución europea. En ambos casos se cubriría la necesidad de que me ocupo ahora, o sea, la de suprimir o limitar la aporía de la que se ha hablado.

En tercer lugar, quisiera referirme a la solución de posibles conflictos de competencias entre la Unión y uno varios Estados miembros por entender éstos que la Unión ha sobrepasado el ámbito atribuido por el tratado. Es decir, si se considera que el Derecho comunitario entra en el terreno de la incompetencia. Es la hipótesis que contempla la Sentencia alemana que se acaba de ver. El problema es: ¿Quién es el habilitado para anular una norma comunitaria incompetente?

A mi juicio puede resultar sorprendente el hecho de que una organización como la Unión Europea, de naturaleza internacional, como ya se ha visto, sea la encargada de delimitar en el caso concreto el alcance de las competencias que se le han atribuido. Pero así es en realidad de modo que esta función la realiza el Tribunal de Justicia. Tampoco sería la mejor solución la propuesta por el Tribunal Constitucional alemán que reclama para sí este posible control del límite de la competencia comunitaria en asuntos que afecten a Alemania. Esta solución no resolvería el problema, al menos con su sola actuación. La aporía se plantearía entonces no entre una norma o sentencia comunitaria y la Constitución alemana sino entre esa misma norma o sentencia comunitaria y la sentencia del Tribunal Constitucional alemán.

En este sentido, quizás le falta a la integración europea un nuevo órgano jurisdiccional encargado exclusivamente de resolver posibles conflictos de competencia entre la Unión y los Estados miembros. En el estadio actual de la integración, y en ausencia de una Constitución europea, este posible órgano jurisdiccional debería tener un claro carácter interestatal y podría estar integrado por miembros de los Tribunales Constitucionales, donde los haya, y de los Tribunales Supremos en los demás casos.

\subsection{Los Estados MiEmbros de LA Unión MANTIENEN SU SOBERANíA}

Creo que después de todo lo que se ha dicho hasta ahora, puede afirmarse que el Estado miembro de la Unión Europea sigue siendo un Estado soberano. Cierto es que a ese Estado soberano el ejercicio de los poderes que derivan de su soberanía, en realidad, todos los que un Estado ejerce, puede resultarle más complicado que a un Estado no comprometido en una organización como la Unión Europea.

A partir del momento en que el Estado se convierte en comunitario, las competencias que se han encomendado a la Unión escapan a su decisión autónoma y pasan a ser ejercidas por las instituciones comunitarias. El Estado, entonces, y respecto de esas competencias, deja de ser la instancia decisoria suprema, que pasa a residir en la Unión. Se trata, como puede verse de una limitación importante en el ejercicio de su soberanía. Lo que antes era desde un punto de vista jurídico una decisión que dependía exclusivamente del propio Estado ha pasado a ser una mera participación en la decisión en el seno del Consejo de Ministros de la Unión al lado de los demás Estados miembros.

Pero esta situación nueva supone únicamente una limitación en el ejercicio de ciertos 
Constitucional alemán reclame para sí el control sobre el respeto de las instituciones comunitarias a la distribución de competencias que hasta este momento ostenta el Tribunal de Justicia comunitario.

En general, dudo que las reformas constitucionales sean eficaces para establecer límites a la integración europea. Nadie duda del importante sentido simbólico que podrían acarrear. Pero, no sé muy bien para qué servirían en relación con el problema de la aporía que se nos plantea porque, realmente, las instituciones comunitarias no tienen porqué atender las indicaciones precisas de una Constitución nacional. La única virtualidad que veo a estas reformas o, más en general, a los límites constitucionales, expresos o no, frente a la integración, es que se impide al Estado admitir un Tratado comunitario que contenga estipulaciones contrarias a la Constitución. Pero esta afirmación nos lleva a caer en la cuenta de que el texto decisivo para precisar las competencias de la Unión Europea no es la Constitución nacional sino la norma superior comunitaria, sea ésta un Tratado o una Constitución europea.

En cuanto a la necesidad apuntada por A. López Basaguren en el sentido que por vía de interpretación los Tribunales nacionales y el comunitario traten de crear reglas que hagan compatibles la vida de ambos ordenamientos, me parece una solución que a veces puede ser absolutamente necesaria. Pero esa solución, si efectivamente se produce, debería considerarse provisional en el sentido de que decisiones de este tipo, a mi juicio, no deben depender de los jueces sino de nuestros representantes políticos. Por tanto, una vez establecida la regla necesaria, ésta debería plasmarse cuanto antes, modificarse o suprimirse en el texto normativo correspondiente.

A partir de las consideraciones anteriores y consciente de las dificultades que presenta el problema, voy a dar algunas opiniones que podrían ser revisadas tras un estudio más detenido del tema.

En primer lugar, creo que es necesario introducir el matiz del principio de competencia en la regla de la primacía. Es decir, sólo cabe hablar de la primacía del Derecho comunitario en las materias de su competencia, en un espacio concreto definido por la norma superior comunitaria. Si el Derecho comunitario va más allá, entra en el terreno de la incompetencia.

En segundo lugar, es necesario un sistema de distribución de competencias dotado de la máxima claridad y precisión posible. Naturalmente, si la distribución de competencias es deficiente, es muy difícil poner límite a ese Derecho que goza de primacía sobre el interno. Y puede nacer la aporía. En cambio, con un sistema lo más claro posible, es más difícil que se produzca tal aporía o contradicción porque, en teoría, al menos, no hay coincidencia en las materias que deben ser reguladas por uno u otro ordenamiento. Y si se propone una coincidencia de ambos ordenamientos en la materia a regular, debe proponerse al mismo tiempo la regla que resuelva el posible conflicto entre normas válidas y contradictorias. Se trata, por ejemplo, de la primacía del Derecho federal previsto en la Constitución alemana (art. 31) y ahora en el proyecto de Constitución europea (art. 10.1) que encajan perfectamente con las previsiones de concurrencia perfecta que se dan tanto en la Ley Fundamental (art. 72) como en el Proyecto de Constitución Europea (art. 11.2). En definitiva, debemos ver la a la Unión Europea como una formación federal y a la luz de los principios federales. En este caso, a la luz del principio de competencia, el más importante desde el punto de vista jurídico.

Se impone, por tanto, establecer una norma superior comunitaria lo más clara y pre- 
y no en un Tratado.

\section{LA CONSTITUCIÓN EUROPEA}

\subsection{Proyectos históricos y objetivos perseguidos}

En los primeros años de las Comunidades Europeas las distintas Asambleas parlamentarias de las tres Comunidades y después el Parlamento Europeo estaban formados, como se sabe, por delegados de los Parlamentos de los Estados miembros. Esta situación se mantuvo hasta 1979 en que tuvo lugar por primera vez la elección del Parlamento Europeo por sufragio universal directo tal como lo conocemos hoy.

A partir de este momento irrumpe en el seno de las Comunidades Europeas un órgano democrático, el Parlamento, formado a partir del voto directo de los pueblos de los Estados miembros. Todos sabemos que históricamente la democratización de las Asambleas legislativas las ha convertido en el núcleo de la decisión política del Estado en detrimento del Monarca.

En las Comunidades Europeas el nuevo Parlamento Europeo, que desempeñaba una mera función consultiva en la adopción de la legislación comunitaria, comenzó a constituir un nuevo motor autónomo dentro del propio sistema y pronto comenzaron las lógicas exigencias de adecuación del origen democrático del Parlamento con su lugar en el procedimiento legislativo.

En este contexto de reivindicación del Parlamento europeo de un lugar y una condición apropiados a su origen democrático, surge de su seno lo que se suele llamar el primer proyecto de Constitución europea o proyecto Spinelli. El Proyecto, de febrero de 1984, que en realidad era y se llamaba «Proyecto de Tratado que instituye la Unión Europea», entrañaba cambios sustanciales en varios puntos.

Modificaba el procedimiento legislativo ya que se declaraba que sería ejercido conjuntamente por el Parlamento y el Consejo. Y en varios momentos se habla de la autoridad legislativa de la Comunidad, integrada por dos ramas, el Parlamento Europeo y el Consejo. Asimismo, se preveía que el Consejo adoptaría ordinariamente sus decisiones por mayoría simple, y en casos previstos expresamente, por mayoría absoluta, cualificada o por unanimidad. También se intenta racionalizar el sistema de fuentes de modo que se contempla la ley como el producto de la autoridad legislativa bicameral y el reglamento, como categoría normativa que ha de ser adoptada por la Comisión para la aplicación de la ley.

De todas formas, el Proyecto Spinelli, que preveía su entrada en vigor mediante un tratado internacional, en caso de revisión, exigía también la ratificación de todos los Estados miembros. Es decir, se trataba una vez más de un instrumento normativo internacional y no exactamente de lo que tradicionalmente llamamos Constitución. Esta observación no resta un ápice a los avances integradores y racionalizadores que el Proyecto encerraba.

En 1991, una Resolución del Parlamento Europeo va a poner en marcha la elaboración de un nuevo proyecto que parta del de Spinelli. Este nuevo Proyecto, que recibió el nombre oficial de Proyecto de Constitución de la Unión Europea, fue aprobado por el 
poderes, es decir, en el ejercicio de la soberanía, pero no una limitación de la soberanía como tal que sigue permaneciendo intacta en sus manos.

Por si alguien ha podido pensarlo, está claro que la soberanía no ha pasado a la Unión Europea y la prueba está en que basta que los Estados miembros se pongan de acuerdo unánimemente para que puedan modificar los tratados comunitarios. Ni tampoco cabría que pasaran a la Unión «trozos» de soberanía. El mismo argumento de la reforma de los tratados niega esta posibilidad. Pero además en este último caso, cuesta pensar en la posibilidad de trocear la soberanía ya que estamos ante un concepto absoluto que sólo se puede concebir si es único en un determinado espacio. No hay soberanías relativas en un determinado espacio, como tampoco hay dos superlativos absolutos en otro espacio concreto.

Por último, la prueba definitiva de que los Estados conservan su soberanía está en el hecho de que, en un determinado momento, pueden abandonar la Unión y dejar de estar sometidos a los compromisos con ella.

El hecho de que la soberanía resida en los Estados miembros de la Unión es determinante para situar a esta organización en el mundo internacional. No obstante, decir de la Unión europea que es una organización internacional es dar un dato fundamental para determinar su naturaleza pero es decir muy poco dada la riqueza y complejidad organizativa que encierra. Y, al decir esto, de acuerdo con el objeto de este trabajo, me limito sólo a los aspectos jurídicos, dejando a un lado otros muchos que se podrían considerar.

En este sentido, se han hecho esfuerzos muy interesantes por matizar más la mera calificación de organización internacional referida a la Unión europea. Ya desde el principio se habló de las Comunidades como organizaciones internacionales de integración, frente a las de cooperación (G. Van Der Meersch). Incluso el Tribunal Constitucional alemán, en la Sentencia citada sobre el Tratado de la Unión Europea, se refiere a una «Unión de Estados» que entronca con una clasificación clásica de las diferentes formas que presentan los Estados en procesos de colaboración, asociación o integración. Finalmente, se ha hablado de la Unión Europea como de una versión moderna de la Confederación cuyo elemento de distinción respecto de las antiguas residiría en el hecho de que no sólo quedarían involucrados los Estados sino también los individuos de esos Estados (A. La Pergola).

De todas formas, la Unión Europea de hoy dista mucho de las primeras Comunidades Europeas de los primeros años. ¿Se podría mantener que también ha podido variar la naturaleza de la organización?

A mi juicio, desde el punto de vista jurídico, creo que el cambio más importante que ha sucedido en la organización comunitaria ha sido el de la elección del Parlamento Europeo por sufragio universal directo y la posterior y lógica incorporación de esta institución con capacidad de decisión al procedimiento legislativo comunitario (codecisión). Este hecho está suponiendo una sacudida muy importante a la antigua forma de decisión exclusiva por parte del Consejo de Ministros y, en definitiva, la asociación de los pueblos europeos a las decisiones comunitarias por medio de sus representantes en el Parlamento Europeo. Y supone también, y no es lo de menos, el nacimiento de una fuente autónoma, comunitaria, de legitimidad en relación con el Derecho comunitario derivado. Esta nueva situación merecería, sin duda, una nueva calificación de la Unión Europea en relación con su organización interna. Pero no afecta a su calidad de organización internacional que solamente podría modificarse en función de la ubicación de la soberanía de modo que ésta pasara de los Estados a la organización y que su base jurídica residiera en una Constitución 
to jurídico estatal.

Este concepto, que antes me ha permitido no admitir como propiamente constitucionales a los Tratados comunitarios, ahora me lleva a no admitir tampoco como verdadera Constitución la que algunos autores han propuesto como posible modelo para la Unión Europea, es decir, una «Constitución confederal». Tal como entiendo la Constitución y la confederación, ambos conceptos no caben juntos ya que son antitéticos. La Constitución es la expresión de un poder constituyente, o sea, soberano y la Confederación es una organización de diferentes Estados soberanos. Partiendo, por tanto, de los conceptos que asumo, no cabe hablar de esta solución para la Unión Europea.

El poder constituyente que está en la base de la Constitución es el pueblo del Estado de que se trate. En el caso de una hipotética Constitución Europea sería el «pueblo europeo». Por tanto, sólo tendremos una Constitución europea cuando ésta haya sido hecha, ratificada, por el pueblo europeo. Llegados a este punto concerniente al pueblo europeo, y siempre se llega a él tarde o temprano si hablamos de una Constitución, junto con tratamientos a mi juicio enteramente correctos (F. Rubio Llorente, J. Ruipérez Alamillo), se suele encontrar la simple respuesta de que el pueblo europeo no existe y, por tanto, no cabe hacer una Constitución.

Una afirmación de este tipo está hecha probablemente desde la intuición personal a partir de alguno o algunos elementos de carácter social que se consideren necesarios para constituir un pueblo. Pero esa visión no es determinante para un jurista. La visión que nos interesa de modo decisivo, especialmente en democracia, es la jurídica. Sólo a través de la certeza que nos da el Derecho conocemos la existencia de una manifestación democrática de querer constituir un pueblo. $\mathrm{Y}$ un grupo humano se constituye jurídicamente como pueblo cuando deja constancia de su voluntad mediante un acto jurídico. Por tanto, no es imprescindible la existencia de un pueblo para hacer una Constitución. Es al revés, en democracia la Constitución es el instrumento por el que una sociedad más o menos homogénea expresa su voluntad de constituirse en pueblo (o de continuar existiendo) y se constituye como tal. En el caso de Europa, la aprobación de una Constitución europea supondría que los pueblos soberanos de los Estados miembros se diluirían en un nuevo pueblo europeo que, a partir de ese momento, sería el único soberano.

A los juristas formados en el marco tradicional del Estado soberano y con la consecuente visión «estatomorfista» o «estatocéntrica», como somos todos nosotros, nos cuadrarían perfectamente todas las cuentas si se hiciera una Constitución Europea. Sería la continuación del esquema jurídico monista desde que nació el Estado que justamente acabó con un esquema pluralista conocido como «poliarquía medieval». La Unión Europea, en su estadio actual, supone hasta cierto punto la vuelta a una nueva poliarquía.

La Constitución europea sería pues la solución lógica. Se acabarían las discusiones acerca de la primacía absoluta del Derecho comunitario puesto que se podría recurrir a la norma suprema de la Constitución. Unicamente, si el Derecho comunitario, en contra de la distribución de competencias adoptada, fuera contradictorio con alguna norma nacional podría plantearse un conflicto de competencias o algún procedimiento destinado a la función de salvaguardia de tal distribución. Es decir, todo quedaría resuelto en el ámbito del ordenamiento comunitario dotado de una norma suprema y, en definitiva, soberana.

Pero los juristas no somos los protagonistas de este proceso. Lo son los representantes de los pueblos europeos y, en definitiva, los pueblos. Y haría falta que éstos quisieran dar ese paso. 
Pleno del Parlamento el 10 de febrero de 1994.

En muchos momentos sigue los pasos del Proyecto Spinelli aunque, como vamos a ver, hay un cambio fundamental en lo que concierne a la revisión de lo que ya puede llamarse Constitución. En el sistema de fuentes, el Proyecto prevé las que llama Leyes constitucionales que tienen una función concreta: modificar o completar la Constitución. Estas leyes se aprueban por la mayoría de $2 / 3$ de los miembros que integran el Parlamento Europeo y por una mayoría muy alta del Consejo.

Es decir, aquí sí que nos encontramos con un instrumento que, una vez aprobado por los Estados integrantes de la Unión Europea de acuerdo con los sistemas internacionales tradicionales, ya no va a exigir la unanimidad para su posible reforma, por lo que entra dentro de lo que podemos denominar una Constitución federal. La intensidad del carácter federal o, si se prefiere, de la integración, será mayor o menor, pero se ha roto la regla de la unanimidad.

Algunas ideas de ambos proyectos han sido parcialmente puestas en práctica por el Tratado de Maastricht y posteriormente, con más intensidad, por el de Amsterdam, de 2 de noviembre de 1997. Por ejemplo, la codecisión en materia legislativa entre el Parlamento y el Consejo.

Como ya he indicado antes, detrás de los proyectos de origen parlamentario estaba fundamentalmente el deseo de asociar a la Cámara elegida al procedimiento legislativo de un modo determinante mediante la codecisión con el Consejo de Ministros y zanjar así el llamado déficit democrático de la Comunidad.

Pero el Parlamento buscaba también una racionalización del complejo sistema comunitario.

Atendiendo a distintas fuentes (consideraciones del propio Parlamento, «Informe Dehaene», presentado el 18 de octubre de 1999 y de diversos autores) se pueden sintetizar en dos los objetivos que se persiguen cuando se reclama una Constitución europea.

Ante todo, se trataría de conseguir una racionalización del sistema institucional comunitario y de sus procesos de decisión a fin de hacerlo más accesible para los ciudadanos. La tradicional ausencia de transparencia del sistema, que resulta ajeno a los ciudadanos, afecta a su legitimidad. Además, en tales circunstancias de opacidad resulta muy difícil el control de sus actores políticos. El Informe Dehaene es muy claro al respecto y sus críticas apuntan tanto al Consejo, como a la Comisión o al propio Parlamento Europeo.

En segundo lugar, se trataría de intentar romper la contradicción entre la supremacía de las Constituciones nacionales y la primacía del Derecho comunitario. Para ello habría que lograr un texto europeo que realizara con la mayor perfección posible la atribución de competencias a la Unión Europea. Además, ese texto tendría que tener carácter constitucional para que se pudiera imponer sin ninguna duda sobre las Constituciones nacionales.

\subsection{Dificultades PARA HACER UNA CONSTITUCIÓN EUROPEA}

Antes de entrar en el tratamiento concreto de este apartado, creo conveniente remitir al lector a la idea de Constitución que he expresado anteriormente y que se puede resumir en que se trata de un texto normativo hecho por el poder constituyente soberano y, por lo tanto, que se impone como norma fundamental dotada de supremacía en un ordenamien- 
La Unión Europea constituye un dato ineludible para los europeos. Pero, de todas formas, que la integración europea sea necesaria, ineludible, no significa que su articulación institucional deba desembocar necesariamente en una unidad política regida por una Constitución, como los Estados Unidos de América o nuestros Estados nacionales clásicos. Como he dicho en otra ocasión, creo que mientras la integración europea es necesaria, una Constitución europea es contingente.

El camino para hacer más profunda esa integración es el de enriquecer los elementos comunes ya conseguidos. Es el Estado comunitario del que ya se ha hablado anteriormente. Es la propia Unión Europea que, a pesar de problemas evidentes internos y de política exterior, constituye una organización con cada vez mayor influencia tanto en la vida de los ciudadanos europeos como en el mundo en general.

Estos elementos comunes a los europeos, que hacen nacer sin duda un sentimiento común de solidaridad, de pertenencia a un espacio más amplio que el del respectivo Estado del que uno es ciudadano, podrán quizás un día convertirse en elementos materiales fundamentales para una Constitución Europea. O tal vez no. Al menos por ahora, no creo que sea tan importante el asunto.

\subsection{El ACTUAL PROYECTO DE CONSTITUCiÓN EUROPEA}

Tras una rápida lectura del Proyecto de Tratado por el que se instituye una Constitución para Europa, adoptado por la Convención Europea el 13 de junio y el 10 de julio de 2003, quiero ahora tratar de ver en qué medida el proyecto responde a los problemas que se han planteado en este trabajo.

A mi juicio, creo que el Proyecto contiene importantes virtudes y, al menos, un defecto también importante.

Empiezo por lo que considero un defecto. El proyecto llama Constitución a lo que realmente no lo es. Cuando el Proyecto regula el proceso para su adopción (Art. IV-8), exige la ratificación «por las Altas Partes Contratantes, de conformidad con sus respectivas normas constitucionales». Está describiendo, por tanto, un tratado. Ciertamente, ese tratado adoptado por todas las partes contratantes podría contener en su seno, tal como parece dar a entender su propio título (Tratado por el que se instituye una Constitución para Europa) un texto que podría convertirse en Constitución. Pero, cuando se observa el procedimiento de reforma del Tratado (Artículo IV-7) se puede ver que, a pesar de que en el procedimiento intervienen en diversos momentos instituciones comunitarias, lo cierto es que tanto la iniciativa como especialmente, la decisión final, están en manos de los Estados miembros. Y en este último caso se exige la unanimidad de los Estados y «las enmiendas entrarán en vigor después de haber sido ratificadas por todos los Estados miembros, de conformidad con sus respectivas normas constitucionales». Estamos por tanto en presencia de un tratado similar a los que hasta ahora han regido la construcción comunitaria.

Incluso parece advertirse que la propia Convención que hace el proyecto no se atreve a sacar todas las conclusiones del nombre de Constitución que atribuye al contenido del Tratado. Y así, en el artículo III-270, en el que se contempla la función del Tribunal de Justicia de velar por la adecuación del Derecho derivado al primario, dice expresamente que «controlará la legalidad de las leyes y las leyes marco europeas». De esta manera, no da el 
Y no es fácil que esto suceda. La soberanía es un símbolo muy acendrado en los pueblos europeos. Y es posible que la sola discusión acerca de la cesión de la soberanía provocara situaciones difíciles en la mayoría de los Estados miembros. El fracaso de los diferentes proyectos parlamentarios que no han salido adelante, especialmente el de 1994, que encerraba un auténtico proyecto de Constitución, son una muestra de ello. Y esto es lo que parece deducirse igualmente del Informe Dehaene y de otro documento de la Comisión, de 10 de noviembre de 1999, que lleva por nombre «Adaptar las instituciones para lograr una ampliación satisfactoria». Y también es una prueba de ello el reciente texto conocido como Proyecto de Constitución Europea que, como veremos, no contempla el nacimiento de una Unión Europea soberana, sino que la soberanía queda donde ha estado siempre, es decir, en los Estados miembros.

En esta situación es preciso volver de nuevo la vista hacia el instrumento del Tratado. Mediante él se han conseguido las cotas de integración alcanzadas hasta hoy y cabe pensar que se puede seguir consiguiendo nuevos avances.

En relación con los dos objetivos que se aducen fundamentalmente para dar el paso hacia una Constitución Europea, ambos podrían ser abordados por la vía del Tratado.

Un tratado podría conseguir con igual capacidad que una Constitución una racionalización y mayor transparencia de la organización y procedimientos comunitarios. No creo que quepa duda al respecto.

En cuanto a la solución de la contradicción entre Constitución interna y Tratados, hay que admitir que una Constitución europea lograría zanjar el asunto ya que implicaría reglas jerárquicas adecuadas relativas a todos los ordenamientos (o, si se prefiere, subordenamientos) afectados y seguramente organizaría un sistema de control de constitucionalidad como los que existen en varios Estados miembros, así como los medios para plantear y solucionar conflictos de competencias entre la Unión y los Estados. Pero, de todas formas, un tratado puede conseguir la misma perfección que una Constitución en la distribución de competencias, lograr fijar reglas precisas para las relaciones interordinamentales e incluso la organización y el procedimiento adecuados para zanjar posibles conflictos de competencia, como ya se ha apuntado antes. En una palabra, mediante la vía tradicional del tratado se puede conseguir paliar en gran parte el problema de la contradicción apuntada.

Por último, es posible hacer que los Tratados o Tratado comunitario funcionen de modo parecido, es decir, ofrezcan un grado de garantía parecido al de una Constitución. El camino nos lo ha enseñado el Tribunal Constitucional alemán al admitir la categoría de «garantía equiparable» del ordenamiento comunitario al alemán en lo concerniente a los Derechos fundamentales y a la democracia. En definitiva, se puede organizar un Tratado que sin tener la cualidad formal de Constitución funcione de modo similar. La garantía última estará en el propio Tratado que, puesto que es objeto de consenso entre los Estados, es una norma rígida y superior en su ámbito a las demás normas. La rigidez le viene del pacto unánime de los Estados miembros que es necesario para introducirla en el ordenamiento. El carácter superior en su ámbito procede de su inicial acomodo con las Constituciones de cada uno de los Estados miembros y del principio de competencia que ha debido presidido su redacción. Es capaz, por tanto, de ejercer una función similar a la de una Constitución, pero sin la obligación de dar el paso traumático de tener que suprimir las soberanías de los pueblos de los Estados europeos. En definitiva, quizá se puedan hacer las cosas de otra manera a como los juristas las hemos aprendido a hacer en el marco de nuestros bien cartesianamente organizados Estados nacionales. 
de construir un futuro común, crea la Unión Europea, a la que los Estados miembros confieren competencias para alcanzar sus objetivos comunes». La Unión Europea se nos presenta así como un instrumento de quienes la crean. Un instrumento al que se le encomiendan tareas concretas o competencias a fin de que sus creadores logren alcanzar sus objetivos comunes. Esto es hoy la Unión Europea. Una Unión de Estados soberanos.

Debe resaltarse también la primera afirmación contenida en el Art. 5.1: «La Unión respetará la identidad nacional de los Estados miembros, inherente a las estructuras fundamentales políticas y constitucionales de éstos [...]». Aquí se encuentra, a mi juicio, una rectificación de los autores a la pretensión inicial del Tribunal de Justicia de que el Derecho comunitario derivado primaba sobre las Constituciones de los Estados miembros. Los autores del Proyecto responden con el deber de la Unión de respetar las estructuras fundamentales políticas y constitucionales de los Estados. Es decir, en nuestros esquemas continentales, la Constitución. Es una manifestación clara de unos límites que se establecen en relación con la actividad de la Unión.

Otro aspecto que resulta aclarado definitivamente por el proyecto se refiere a la posibilidad de que un Estado miembro se retire voluntariamente de la Unión. Así se recoge en el artículo 59.1. «Todo Estado miembro podrá decidir, de conformidad con sus normas constitucionales, retirarse de la Unión Europea.»

Como ya he señalado anteriormente, este artículo no innova el ordenamiento jurídico de la Unión respecto de lo que antes sucedía. Antes esta posibilidad existía en virtud de la denuncia de los tratados según el Derecho internacional general. Sin embargo, se trata de una aclaración importante. El hecho de que la regla se haga explícita en la propia norma superior comunitaria puede evitar suspicacias infundadas y tesis doctrinales difíciles como las que han defendido la imposibilidad de abandonar la UE.

Alguien podría pensar que se trata de un Derecho de secesión. Pero es una situación muy distinta porque quienes integran la Unión Europea son Estados soberanos. Es decir, nos movemos en el mundo internacional y la posibilidad de separarse, ahora recordada dentro del propio proyecto, se desprende de ese mismo Derecho internacional.

Tampoco podría identificarse por motivos parecidos con el Derecho de autodeterminación.

En todo caso, se trata de una manifestación de que la soberanía permanece en los Estados, limitada únicamente en cuanto a su ejercicio por los compromisos adquiridos por tal Estado.

Es un acierto, a mi juicio, que el proyecto acabe también de un plumazo con las discusiones acerca del significado de la duración indefinida de la Unión (Artículo IV-9). Duración indefinida, sí, pero sólo para los Estados que así lo quieran.

El proyecto hace también un esfuerzo notable por clarificar la distribución de competencias entre los Estados y la Unión europea. Además de la concreción de muchos supuestos singulares de competencias que se atribuyen a la Unión y que están presentes a lo largo del proyecto, éste contiene también un intento de tratamiento general del problema. Distingue, así, entre competencias exclusivas de la Unión (art. 12) y ámbitos de competencias compartidas (art. 13). Previamente (art. 11) el Proyecto describe las facultades inherentes a las diferentes categorías de competencias. Se trata de un esfuerzo de racionalización necesario y ajustado a la realidad de la Unión.

Previamente al tratamiento genérico de las competencias, el proyecto recoge el princi- 
paso de hablar de control de constitucionalidad como convendría si estuviéramos en presencia de una Constitución. De hecho se mantiene la misma redacción que la que daba el art. 230 del tratado de la Comunidad Económica Europea.

Y creo que esto es un error. La Constitución supone, entre otras cosas, un símbolo político demasiado importante como para restarle valor con usos indebidos. Ciertamente, no se ha acertado dando el nombre de Constitución europea a lo que no es sino un Tratado internacional. Además, el uso indebido de esta palabra puede arrastrar hacia una confusión permanente sobre cuestiones decisivas acerca de la ubicación de la soberanía o del alcance de la primacía del Derecho comunitario, por ejemplo. A mi no me cabe ninguna duda de que la soberanía sigue residiendo en cada uno de los Estados miembros, y el propio Proyecto nos da pruebas inequívocas de ello, como se verá más tarde, pero la semilla la confusión ya está sembrada.

Pero, al mismo tiempo que critico el «nombre» utilizado para llamar a la «cosa» que han creado, reconozco sin paliativos la gran virtud de la Convención de haber mantenido la trayectoria práctica de quienes a lo largo de los años han ido haciendo lo que hoy constituye la Unión Europea y de no haber intentado dar saltos en el vacío. Me refiero al hecho de que, a pesar del nombre, la cosa, o sea, la Norma Superior Comunitaria sigue siendo un Tratado. Un proyecto de una Constitución de verdad se hubiera convertido con mucha seguridad en un motivo de polémicas dramáticas y estériles. Ya me he referido antes al asunto. El Tratado, en este caso, es un instrumento eficaz que encierra una gran dosis de cautela y prudencia.

Otra virtud del proyecto de norma superior comunitaria, que comparte con los Tratados de Maastricht y siguientes, es el hecho de que los Estados son quienes adoptan decisiones estructurales, fundamentales sobre la Unión Europea. Es decir, toman la iniciativa sobre los modos de la integración comunitaria. Lo hicieron, evidentemente, en los primeros Tratados de París y de Roma. Pero es difícil que los autores de una norma sean capaces de prever todas las situaciones con las que esa norma se va a encontrar. Por ello, normalmente hay momentos en que corresponde al juez no innovar el ordenamiento jurídico pero sí hacer explícitos principios que se deducen del mismo. Esta operación la realiza mediante decisiones aplicadas a un caso concreto pero que tienen una incidencia general sobre toda la estructura ordinamental. Un ejemplo claro es la declaración de la primacía del Derecho comunitario. Como este ejemplo pueden citarse otros.

En estos casos, hay veces en que la actividad del Tribunal de Justicia puede ser acorde con lo que los Estados pueden aceptar. También puede haber decisiones del Tribunal que, en general, los Estados querrían modificar o, al menos, aclarar. Mediante su actividad normativa de creación de una nueva norma superior comunitaria, los Estados pueden dar por sentados ciertos valores afirmados por la jurisprudencia, reafirmándolos o aceptándolos implícitamente. Y también podrían matizar o, incluso, negar algunos otros.

Lo importante de todo ello es que los Estados, en tanto que expresión jurídica de los pueblos soberanos europeos, toman la iniciativa política en la integración comunitaria. Iniciativa que sólo puede corresponder a los Tribunales de forma interina hasta que el legislador, que representa al pueblo o pueblos, hable de nuevo.

También debe colocarse en el haber del proyecto la claridad con que el Art. 1.1 del texto nos da una pista segura sobre la naturaleza de la Unión Europea. Dice así: «La presente Constitución, que nace de la voluntad de los ciudadanos y de los Estados de Europa 
de procesos corresponde al Tribunal de Justicia comunitario convertimos a esta institución en la poseedora de la última palabra sobre el conflicto de competencias entre el acto comunitario y los ordenamientos nacionales.

En definitiva, en este terreno, las cosas se han dejado como estaban. El Tribunal de Justicia va a tener la libertad que siempre ha tenido para decidir sobre los límites de la integración europea. Es decir, se ha avanzado conceptualmente en la técnica de la distribución de las competencias pero no se ha establecido una vía específica para esclarecer la atribución de la competencia en caso de conflicto.

Y es dudoso que el Tribunal de Justicia, dada su situación en el marco de la Unión, sea el instrumento más idóneo para llevar a cabo esta función.

Desde los Tratados de París y de Roma hasta hoy se ha encomendado al Tribunal la garantía del respeto del Derecho en la interpretación de los Tratados (art. 31 Tratado CECA y 220 CEE). Y el proyecto de Constitución Europea sigue manteniendo esta función como la ordinaria del Tribunal de Justicia. Así lo hace en el art. 28.1: «El Tribunal de Justicia [...] Garantizará el respeto del Derecho en la interpretación y aplicación de la Constitución». Es decir, se observa que el objeto central de la visión del Tribunal de Justicia es el Tratado o Norma Superior Comunitaria. Y parece conveniente que al ejercer la función de que se habla aquí no sólo es necesario tener en cuenta esa Norma Superior Comunitaria sino también las Constituciones de los Estados miembros. De todas formas, esta objeción apenas tendría importancia si estuviéramos ante una auténtica Constitución europea. En este caso, el Tribunal superior definido por esa Constitución, fruto de un poder constituyente europeo, sería el indicado, sin duda, para dar solución a estos problemas, como sucede en los Estados federales. Pero, como se sabe, no estamos ante una Constitución.

Por ello todavía adquiere mucha más importancia la objeción siguiente. El Tribunal aún creado con una intensa intervención de los Estados miembros, es una institución comunitaria. Esto significa que estamos ante una Institución de la organización creada por los Estados para alcanzar sus objetivos comunes (Art. 1 del Proyecto). En el vértice del ordenamiento jurídico de cada uno de esos Estados se encuentra una Constitución que nunca podría ser contradicha por las normas o decisiones jurisprudenciales del instrumento creado a partir de las habilitaciones permitidas por esas Constituciones.

Parece, por tanto, que ni por las funciones que se le encomiendan ni, sobre todo, por su situación estructural, el Tribunal de Justicia sea el mejor juez en los posibles conflictos de competencias entre los Estados y la propia Unión.

Hacia el futuro, y mientras no estemos ante una verdadera Constitución europea, quizás fuera necesario indagar sobre la posibilidad de un nuevo órgano interestatal como el que he señalado anteriormente. 
pio de la primacía del Derecho comunitario y le imprime un sello particular y fundamental. La jurisprudencia del Tribunal de Justicia, desde la sentencia 6/60, Humblet c. Etat belge, de 16 de diciembre de 1960, pasando por las más conocidas Costa c. ENEL, de 1969, o la Internationale Handelgesellschaft, de 1970, declaró la primacía del Derecho comunitario sobre el Derecho interno. No había matices respecto de esa calificación. Cualquier norma de Derecho comunitario sin limitación en cuanto a la materia a la que se refiriera, gozaba de primacía.

El proyecto, en cambio, se manifiesta de la manera siguiente en el art. 10.1: «La Constitución y el Derecho adoptado por las instituciones de la Unión en el ejercicio de las competencias que le son atribuidas primarán sobre el Derecho de los Estados miembros.» El gran matiz introducido por el proyecto consiste en que el Derecho comunitario queda limitado al «ejercicio de las competencias que le son atribuidas» a la Unión.

Este importantísimo matiz o límite supone en definitiva comenzar a tratar a la Unión Europea con criterios federales. Porque, de esta manera, en lo que concierne a las relaciones entre ordenamiento comunitario y ordenamientos de los Estados miembros se introduce como regla central el principio de competencia. Es decir, y vuelvo al límite establecido por el proyecto, si el Derecho comunitario intenta regular materias sobre las que no se ha atribuido ninguna competencia a la Unión, entra en el terreno de la incompetencia.

Este es un fenómeno perfectamente conocido en España, por citar un ejemplo familiar para mí. Si el Estado o una Comunidad Autónoma regulan materias que están fuera del ámbito de sus competencias, sus actos pueden ser declarados inconstitucionales y nulos por el Tribunal Constitucional mediante el procedimiento del conflicto de competencias.

Un razonamiento de este tipo puede ser perfectamente aplicado a las relaciones entre el ordenamiento jurídico comunitario y los nacionales. Pero es posible que no tengamos los medios adecuados para llevar a la práctica tal razonamiento lógico.

En principio hay que dar por supuesto que el conflicto siempre es posible a pesar de la perfección con que pueda haberse llevado a cabo la distribución de competencias. En la práctica la dificultad para decidir que estamos en el ámbito de una u otra materia puede presentarse en cualquier momento. Por tanto es necesario contar con medios para resolver el conflicto planteado.

El Proyecto no contempla específicamente estos posibles conflictos. Cuando el texto se refiere a la jurisdicción del Tribunal de Justicia comunitario, recoge sin ningún nuevo matiz las causas que ya contemplaba el Tratado de la Comunidad Económica Europea (art. III-270.2): «A tal fin (controlar la legalidad de los actos comunitarios), será competente para pronunciarse sobre los recursos por incompetencia, vicios sustanciales de forma, violación de la Constitución o de cualquier norma jurídica relativa a su ejecución, o desviación de poder». Dada la redacción del precepto y la interpretación histórica que se ha hecho del mismo, la posibilidad para atacar un acto de la Unión, presuntamente viciado de la incompetencia interordinamental de que se habla aquí, no sería debida curiosamente a la causa de la «incompetencia» a la que se refiere el propio precepto. Esta incompetencia siempre se ha entendido en relación con las diferentes funciones reservadas a las distintas Instituciones comunitarias. La causa sería, por tanto, la de la «violación de la Constitución» que es la norma superior comunitaria en la que se ha hecho el reparto de competencias entre los Estados miembros y la Unión.

Es decir, existe una vía procedimental que puede ser utilizada tanto por la Unión como por los Estados miembros. Pero el problema reside en que si la decisión en este tipo 
